\title{
Evolution of cerebrospinal fluid total $\alpha$-synuclein in Parkinson's disease
}

Marthe Gurine Førland ${ }^{\mathrm{a}}$, Annika Öhrfelt ${ }^{\mathrm{b}}$, Ingvild Dalen ${ }^{\mathrm{c}}$, Ole-Bjørn Tysnes ${ }^{\mathrm{d}, \mathrm{e}}$, Kaj Blennow ${ }^{\mathrm{b}, \mathrm{f}}$, Henrik Zetterberg ${ }^{\mathrm{b}, \mathrm{f}, \mathrm{g}, \mathrm{h}}$, Kenn Freddy Pedersen ${ }^{\mathrm{a}, \mathrm{i}}$, Guido Alves ${ }^{\mathrm{a}, \mathrm{i}, \mathrm{j}}$ and Johannes Lange ${ }^{\mathrm{a}, *}$

a: The Norwegian Centre for Movement Disorders, Stavanger University Hospital, PO Box 8100, 4068 Stavanger, Norway

b: Institute of Neuroscience and Physiology, The Sahlgrenska Academy at the University of Gothenburg, Sahlgrenska University Hospital at Mölndal, University of Gothenburg, 43180 Mölndal, Sweden

c: Section of Biostatistics, Department of Research, Stavanger University Hospital, PO Box 8100, 4068 Stavanger, Norway

d: Department of Neurology, Haukeland University Hospital, PO Box 1400, 5021 Bergen, Norway

e: Institute for Clinical Medicine, University of Bergen, PO Box 1400, 5021 Bergen, Norway

f: Clinical Neurochemistry Laboratory, Sahlgrenska University Hospital, 43180 Mölndal, Sweden

g: Department of Molecular Neuroscience, UCL Institute of Neurology, University College London, Gower Street, WC1E 6BT London, UK

h: UK Dementia Research Institute, Box 16, National Hospital for Neurology and Neurosurgery

Queen Square, WC1N 3BG London, UK

i: Department of Neurology, Stavanger University Hospital, PO Box 8100, 4068 Stavanger, Norway

j: Department of Mathematics and Natural Sciences, University of Stavanger, Stavanger, Norway

* Correspondence to: Dr. Johannes Lange, The Norwegian Centre for Movement Disorders, PO Box 8100, N-4068 Stavanger, Norway; +47-5151-5602; johannes.lange@ sus.no

Word Count

\begin{tabular}{ll} 
Abstract & 170 \\
\hline Introduction & 188 \\
Methods & 753 \\
Results & 164 \\
Discussion & 783 \\
Manuscript w/o abstract & 1888 \\
\hline References & 30 \\
Tables & 1 \\
Figures & 2 \\
Supplementary tables & 2
\end{tabular}

Running title: Longitudinal CSF total $\alpha$-synuclein in PD

Key words: $\alpha$-synuclein, cerebrospinal fluid, biomarker, longitudinal measurements, Parkinson's disease.

\section{Conflicts of interest: None}

Financial disclosure: This study was supported by the Western Norway Regional Health Authority [grant 911218 and 911832] and the Norwegian Parkinson's Disease Association.

\section{Abstract}


Introduction: Cerebrospinal fluid (CSF) total $\alpha$-synuclein is considered a potential biomarker for Parkinson's disease (PD), but little is known about the evolution of this marker during the course of the disease. Our objective was to investigate whether CSF total $\alpha$-synuclein concentrations change over time and are associated with motor and cognitive function in PD.

Methods: CSF total $\alpha$-synuclein concentrations were quantified in 56 longitudinally followed PD patients, 27 of whom provided CSF repeatedly 2 and/or 4 years later. Another 18 subjects were included as controls. The samples were analyzed using two independent, validated ELISA methods: our recently developed and validated in-house ELISA and a commercial kit from BioLegend.

Results: CSF total $\alpha$-synuclein levels did not distinguish PD patients from controls, displayed no substantial changes during a period of up to 4 years, and did not predict subsequent motor or cognitive decline. These findings were consistent for both analytical methods.

Conclusion: Our findings do not support the clinical utility of total $\alpha$-synuclein as a single diagnostic or prognostic biomarker in PD. 


\section{Introduction}

Parkinson's disease (PD) is characterized by a remarkable clinical heterogeneity, with substantial variation in disease progression and considerable overlap with other neurodegenerative diseases. Therefore, reliable biomarkers for diagnosis and prognosis of PD are urgently needed. Given the central role of $\alpha$-synuclein aggregation in PD pathogenesis, and because $\alpha$-synuclein levels are a major determinant of its neurotoxicity [1], it is of great interest to investigate the potential of $\alpha$-synuclein as a diagnostic and prognostic biomarker. In this respect, cerebrospinal fluid (CSF) is considered the most promising source. Most, but not all previous studies report a trend towards lower total CSF $\alpha$-synuclein levels in PD compared to controls, though with substantial overlap between diagnostic groups [2]. Only a few studies have reported longitudinal measurements of $\alpha$-synuclein, also with conflicting results [3-6]. Hence, there is a need for additional longitudinal studies to track CSF $\alpha$-synuclein changes during the disease course. We also examined whether total $\alpha$-synuclein concentrations were associated with motor symptoms and neuropsychological performance, and their changes over a period of up to 4 years. In addition, we compared total $\alpha$-synuclein levels of PD patients with those of the controls. 


\section{Materials and methods}

\section{Patients}

All PD patients were enrolled in the Norwegian ParkWest study, an ongoing, prospective, population-based, longitudinal cohort study of PD in Southwestern Norway [7]. Participants included a subset of 56 PD patients (median disease duration 4.8 years), of whom 27 also provided CSF at one or two follow-up visits 2 and 4 years later (Figure 1 and Table 1). The patients were followed prospectively by movement disorders neurologists and fulfilled the National Institute of Neurological Disorders and Stroke [8] and UK Brain Bank [9] diagnostic criteria of PD from study entry to latest visit. Exclusion criteria were secondary parkinsonism and dementia during the first year of motor onset to eliminate patients with dementia with Lewy bodies and other dementias, including Alzheimer's disease. All patients underwent a uniform and standardized examination program at each visit. Disease severity was determined using the Unified Parkinson's Disease Rating Scale (UPDRS) [10] part II and III, and disease stage was evaluated using the Hoehn and Yahr scale (H\&Y) [11]. Cognitive assessments included the Mini-Mental State Examination (MMSE) [12] as a measure of global cognition, and a neuropsychological test battery assessing the following domains: verbal memory (California Verbal Learning Test II [CVLT-II] [13], which included total immediate recall [sum of trials 1-5], short-delay, and long-delay free recall scores), attention (Stroop word reading and color naming) [14], executive functioning (Semantic Verbal Fluency Test [15], and Stroop interference condition) and visuospatial skills (Visual Object and Space Perception battery [VOSP] Silhouettes and Cube subtests) [16]. Severity of depressive symptoms was determined using the Montgomery and Aasberg Depression Rating Scale (MADRS) [17].

\section{Controls}

The control group consisted of 18 subjects without known brain disease, who underwent elective neurological examination or orthopedic surgery at Stavanger University Hospital. 


\section{CSF sample treatment}

CSF collection and sample treatment was conducted according to standardized procedures, as described elsewhere [18]. The CSF samples were centrifuged at 2000g for 10 minutes and frozen in polypropylene tubes at $-80^{\circ} \mathrm{C}$. The samples were subjected to one freeze-thaw event for aliquotation purposes.

\section{$\alpha$-synuclein measurement}

The in-house assay procedures and characteristics have been described in detail previously [19]. In brief, the ELISA assay fulfills the newest validation criteria [20], has a lower limit of quantification of $36.3 \mathrm{pg} / \mathrm{mL}$ and requires less than $15 \mu \mathrm{L}$ CSF. The samples were analyzed in duplicates at the Neuroscience Research Laboratory at Stavanger University Hospital, Norway, with plate-to-plate variation below $6 \%$. An identical set of CSF aliquots were sent to the Clinical Neurochemistry Laboratory at Sahlgrenska University Hospital, Sweden for analysis with the commercial ELISA kit from BioLegend (\#844101; BioLegend, San Diego, USA), with plateto-plate variation below 5\%. There was a high correlation between the two assays $(\rho=0.785$, $P<0.001$ ), which were independent in terms of site, date of analysis and personnel.

\section{Hemoglobin measurement}

To assess possible blood contamination as source of $\alpha$-synuclein, the samples were analyzed for hemoglobin content using a commercial kit (\#E88-135, Bethyl Laboratories, Montgomery, USA). Samples with hemoglobin concentrations above $200 \mu \mathrm{g} / \mathrm{mL}$ were excluded from the study.

\section{Statistical analysis}

Standard curve fitting (4-parameter logistic fit), plotting of calibrator curves and calculation of total $\alpha$-synuclein concentrations were performed using the Meso Scale Discovery Workbench 4.0 software (Meso Scale Discovery, Gaithersburg, USA). Descriptive statistics for continuous 
variables are presented as means for symmetrically distributed data, as geometric means for right skewed, small samples, or otherwise as medians, and with full ranges. Categorical variables are presented with counts and percentages. Between-group comparisons were performed by Mann-Whitney U for continuous variables. Within-patient comparisons were performed by paired samples t-tests for symmetrically distributed differences, or Wilcoxon paired signed rank tests for non-normally distributed data. Possible relationships between CSF total $\alpha$-synuclein and clinical and neuropsychological performance at CSF sampling were assessed by linear regression analysis with adjustment for age and sex. To assess associations between CSF total $\alpha$-synuclein and clinical and neuropsychological measures within 4 years after CSF sampling, we used linear random intercept models with adjustment for age and sex, and with time and CSF total $\alpha$-synuclein included as main effects and with an interaction term, that measures the possible differential effect of levels of $\alpha$-synuclein on progression slopes. Spearman's rank correlation was used to assess strength of association between the two analytical methods. All analyses were conducted using SPSS 23 (IBM, Armonk, USA). Twotailed $P$-values $<0.05$ were considered statistically significant. For multiple comparisons, Bonferroni correction was applied with two-tailed $P$-values $<0.0036$ (14 tests) considered significant.

\section{Research Ethics}

All procedures were approved by The Norwegian Regional Committee for Medical and Health Research Ethics, and signed written informed consent was obtained from all participants. 


\section{Results}

There was no significant difference in CSF total $\alpha$-synuclein concentrations between PD patients $(\mathrm{N}=56,55 \%$ males, median age 69.6$)$ and controls $(\mathrm{N}=18,50 \%$ males, median age 67.7), as measured by the in-house assay (469 [178-1333] pg/ml vs. 424 [175-852] pg/ml; $P=0.392)$, and the BioLegend kit (677 [259-1723] pg/ml vs. 747 [306-1623] pg/ml; $P=0.366)$

(Figure 2A and B). In the PD patients, CSF levels were not significantly associated with clinical or cognitive performance cross-sectionally (Table s1), nor did they predict the rate of motor or cognitive decline longitudinally during up to 4 years of follow-up (Table s2), after correction for multiple testing. A separate analysis excluding patients with PD dementia or depression did not alter the overall result (data not shown). Finally, we found no consistent pattern nor any statistically significant changes in CSF total $\alpha$-synuclein concentrations during 4 years of follow-up ( $\mathrm{N}=27$, all $P>0.1$; Table 1, Figure $2 \mathrm{~A}$ and $\mathrm{B}$ ), although some variation over time was observed in a few individuals (Figure 2C and D). 


\section{Discussion}

In this study of PD patients recruited from a prospective, population-based longitudinal study, we found that total CSF $\alpha$-synuclein levels, measured by two independent analytical methods, 1) did not differ from controls, adding to the list of studies reporting comparable levels between PD and controls [2]; 2) displayed no significant changes during a period of up to 4 years, and 3) did not predict the rate of motor, cognitive or neuropsychological decline, in agreement with previous studies [2]. Overall, these findings do not support the clinical utility of total $\alpha$ synuclein as a single diagnostic or prognostic biomarker in PD.

In recent years, CSF total $\alpha$-synuclein and other $\alpha$-synuclein species have emerged as potential diagnostic and prognostic biomarkers in PD [2]. However, while numerous cross-sectional studies have investigated the diagnostic value of total $\alpha$-synuclein, very few longitudinal studies have been conducted to explore changes of CSF total $\alpha$-synuclein over time, and with inconsistent findings. Two studies utilizing data from the DATATOP cohort found either decreasing [3] or increasing [4] $\alpha$-synuclein levels over a 2-year period. These apparently contradicting findings might be explained by differences in pre-analytical factors or detection methods [4]. Another study, using the Swedish BioFINDER cohort, found a minor increase at the group level in CSF $\alpha$-synuclein levels over a period of 2 years in PD patients with long $(>5$ years) but not shorter ( $\leq 5$ years) disease duration [5]. Finally, a recent report from the PPMI study revealed no change over a period of $1 / 2$ and 1 year in early PD [6], in line with results from our 4-year longitudinal study.

In Alzheimer's disease, it is well established that the deposition of amyloid-beta into extracellular plaques translates into decreased CSF concentrations of this protein [21]. In PD however, $\alpha$-synuclein aggregates into Lewy bodies intracellularly, making it less clear how these pathological events, and their spread in the brain, influence the dynamics of $\alpha$-synuclein in CSF. Different hypotheses explaining CSF changes of total $\alpha$-synuclein levels in either 
direction have been proposed. A reduction over time could be explained by the sequestration and aggregation of $\alpha$-synuclein in soluble fibrils and Lewy bodies [2], while an increase in levels could result from progressive neuronal damage [22], possibly due to release of $\alpha$ synuclein from degenerating cells. Indeed, some PD studies have shown strong correlations between CSF levels of $\alpha$-synuclein and tau, an acknowledged marker of neuronal degeneration $[23,24]$. Furthermore, a marked increase in levels of $\alpha$-synuclein has been found in disorders with widespread and progressive neurodegeneration, such as Creutzfeldt-Jakob disease and AD [25-29]. A co-occurrence of such contrary events may result in no overall change in total $\alpha$ synuclein levels, as found in our study. Furthermore, Hall et al. [5] hypothesized that the evolution of $\alpha$-synuclein levels in PD might be bimodal with levels decreasing in early disease due to intracellular accumulation, followed by an increase with clinical disease progression reflecting progressive neuronal damage. In our study, CSF was obtained from patients at least 3 years after motor onset, and applying the bimodal hypothesis, patients may already have reached a plateau in the second stage of the disease development. This might explain why there was no further increase in $\alpha$-synuclein levels with continued disease progression. However, more longitudinal studies with multiple sampling covering longer periods are needed to fully clarify this issue.

Another implication of the bimodal hypothesis is that the potential diagnostic value of $\alpha$ synuclein may be offset by individual differences in the neuropathological effects accountable for changes in total $\alpha$-synuclein levels in CSF. Therefore, an approach with $\alpha$-synuclein as part of a biomarker panel, including other species of $\alpha$-synuclein, such as oligomeric- and phosphorylated $\alpha$-synuclein, could be a promising alternative [30].

A limitation of this study is the relatively small sample size, particularly of those with repeated CSF measurements, contributing to wide confidence intervals. In addition, the limited sample size did not allow for subgroup analysis (e.g. after stratification into different endophenotypes). 
Therefore, we cannot exclude the possibility of associations of CSF $\alpha$-synuclein with clinical features in certain PD subgroups. Another limitation, shared by similar studies, is that patients with milder symptoms are possibly more likely to consent to repetitive lumbar puncture, which could result in a potential selection bias. Finally, the lack of other CSF markers than $\alpha$-synuclein (e.g. tau) and the inhomogeneous time intervals between lumbar punctures in those with repeated measures can be considered further limitations of this study. Strengths include the unselected PD cohort, comprehensive, prospective clinical and neuropsychological assessments, and study design with repeated CSF samples over a longer time interval than in previous studies. Finally, we followed well-established and standardized routines for collection and handling of CSF samples, and applied two independent, validated methods to quantify total $\alpha$-synuclein levels with consistent findings. 


\section{Acknowledgements}

The authors thank the patients of the ParkWest study and CSF donors for participating in this study. We are grateful to Åsa Källén for her technical assistance. We would also like to thank the Western Norway Regional Health Authority, the Norwegian Parkinson's Disease Association, the Swedish Research Council, the European Research Council, the Knut and Alice Wallenberg Foundation, the Torsten Söderberg Foundation, the Swedish Brain Foundation, and the Swedish Alzheimer Foundation for financial support. 


\section{Author Contributions}

Marthe Gurine Førland: Conception of experiments, Execution of experimental work, Design and Execution of Statistical Analysis, wrote the first draft of the Manuscript, Review and Critique of the Manuscript

Annika Öhrfelt

Ingvild Dalen

Ole-Bjørn Tysnes:

Kaj Blennow:

Henrik Zetterberg:

Kenn-Freddy Pedersen:

Guido Alves:

Johannes Lange:
Organization of Research Project, Review and Critique of the Manuscript

Statistical Analysis, Review and Critique of the Manuscript

Organization of Research Project, Review and Critique of the Manuscript

Organization of Research Project, Review and Critique of the Manuscript

Organization of Research Project, Review and Critique of the Manuscript

Organization of Research Project, Review and Critique of the Manuscript

Organization of Research Project, Review and Critique of Statistical Analysis and the Manuscript

Conception of experiments, Execution of experimental work, Design and Execution of Statistical Analysis, Review and Critique of the Manuscript 


\section{References}

[1] M. Xilouri, O.R. Brekk, L. Stefanis, alpha-Synuclein and protein degradation systems: a reciprocal relationship, Mol Neurobiol 47(2) (2013) 537-51.

[2] L. Parnetti, C. Cicognola, P. Eusebi, D. Chiasserini, Value of cerebrospinal fluid alpha-synuclein species as biomarker in Parkinson's diagnosis and prognosis, Biomark Med 10(1) (2016) 35-49.

[3] T. Stewart, C. Liu, C. Ginghina, K.C. Cain, P. Auinger, B. Cholerton, M. Shi, J. Zhang, D.I. Parkinson Study Group, Cerebrospinal fluid alpha-synuclein predicts cognitive decline in Parkinson disease progression in the DATATOP cohort, Am J Pathol 184(4) (2014) 966-75.

[4] N.K. Majbour, N.N. Vaikath, P. Eusebi, D. Chiasserini, M. Ardah, S. Varghese, M.E. Haque, T. Tokuda, P. Auinger, P. Calabresi, L. Parnetti, O.M. El-Agnaf, Longitudinal changes in CSF alphasynuclein species reflect Parkinson's disease progression, Mov Disord 31(10) (2016) 1535-1542. [5] S. Hall, Y. Surova, A. Ohrfelt, F.S. Swedish Bio, K. Blennow, H. Zetterberg, O. Hansson, Longitudinal Measurements of Cerebrospinal Fluid Biomarkers in Parkinson's Disease, Mov Disord 31(6) (2016) 898-905.

[6] B. Mollenhauer, C.J. Caspell-Garcia, C.S. Coffey, P. Taylor, L.M. Shaw, J.Q. Trojanowski, A. Singleton, M. Frasier, K. Marek, D. Galasko, I. Parkinson's Progression Marker, Longitudinal CSF biomarkers in patients with early Parkinson disease and healthy controls, Neurology (2017).

[7] G. Alves, B. Muller, K. Herlofson, I. HogenEsch, W. Telstad, D. Aarsland, O.B. Tysnes, J.P. Larsen, g. Norwegian ParkWest study, Incidence of Parkinson's disease in Norway: the Norwegian ParkWest study, J Neurol Neurosurg Psychiatry 80(8) (2009) 851-7.

[8] D.J. Gelb, E. Oliver, S. Gilman, Diagnostic criteria for Parkinson disease, Arch Neurol 56(1) (1999) 33-9.

[9] A.J. Hughes, Y. Ben-Shlomo, S.E. Daniel, A.J. Lees, What features improve the accuracy of clinical diagnosis in Parkinson's disease: a clinicopathologic study. 1992, Neurology 57(10 Suppl 3) (2001) S34-8.

[10] S. Fahn, Recent developments in Parkinson's disease, Raven Pr1986.

[11] M.M. Hoehn, M.D. Yahr, Parkinsonism: onset, progression and mortality, Neurology 17(5) (1967) 427-42.

[12] M.F. Folstein, S.E. Folstein, P.R. McHugh, "Mini-mental state". A practical method for grading the cognitive state of patients for the clinician, J Psychiatr Res 12(3) (1975) 189-98.

[13] D.C. Delis, J.H. Kramer, E. Kaplan, B.A. Ober, Manual for the California Verbal Learning Test, (CVLT-II), San Antonio, TX: The Psychological Corporation ed.2000.

[14] C.J. Golden, S.M. Freshwater, The Stroop Color and Word Test, Wood Dale: Stoelting Co1998. [15] A.L. Benton, K.D. Hamster, Multilingual Aphasia Examination, Iowa City: AJA Associations (1989).

[16] E.K. Warrington, M. James, The Visual Object and Space Perception Battery, Bury St. Edmunds: Thames Valley Test Co (1991).

[17] S.A. Montgomery, M. Asberg, A new depression scale designed to be sensitive to change, The British journal of psychiatry : the journal of mental science 134 (1979) 382-9.

[18] G. Alves, J. Lange, K. Blennow, H. Zetterberg, U. Andreasson, M.G. Forland, O.B. Tysnes, J.P. Larsen, K.F. Pedersen, CSF Abeta42 predicts early-onset dementia in Parkinson disease, Neurology 82(20) (2014) 1784-90.

[19] M.G. Forland, A. Ohrfelt, L.S. Oftedal, O.B. Tysnes, J.P. Larsen, K. Blennow, H. Zetterberg, G. Alves, J. Lange, Validation of a new assay for alpha-synuclein detection in cerebrospinal fluid, Clin Chem Lab Med (2016).

[20] U. Andreasson, A. Perret-Liaudet, L.J. van Waalwijk van Doorn, K. Blennow, D. Chiasserini, S. Engelborghs, T. Fladby, S. Genc, N. Kruse, H.B. Kuiperij, L. Kulic, P. Lewczuk, B. Mollenhauer, B. Mroczko, L. Parnetti, E. Vanmechelen, M.M. Verbeek, B. Winblad, H. Zetterberg, M. Koel-Simmelink, C.E. Teunissen, A Practical Guide to Immunoassay Method Validation, Frontiers in neurology 6 (2015) 179.

[21] K. Blennow, N. Mattsson, M. Scholl, O. Hansson, H. Zetterberg, Amyloid biomarkers in Alzheimer's disease, Trends in pharmacological sciences 36(5) (2015) 297-309. 
[22] A. Lleo, E. Cavedo, L. Parnetti, H. Vanderstichele, S.K. Herukka, N. Andreasen, R. Ghidoni, P. Lewczuk, A. Jeromin, B. Winblad, M. Tsolaki, B. Mroczko, P.J. Visser, I. Santana, P. Svenningsson, K. Blennow, D. Aarsland, J.L. Molinuevo, H. Zetterberg, B. Mollenhauer, Cerebrospinal fluid biomarkers in trials for Alzheimer and Parkinson diseases, Nature reviews. Neurology 11(1) (2015) 41-55.

[23] J.H. Kang, D.J. Irwin, A.S. Chen-Plotkin, A. Siderowf, C. Caspell, C.S. Coffey, T. Waligorska, P. Taylor, S. Pan, M. Frasier, K. Marek, K. Kieburtz, D. Jennings, T. Simuni, C.M. Tanner, A. Singleton, A.W. Toga, S. Chowdhury, B. Mollenhauer, J.Q. Trojanowski, L.M. Shaw, I. Parkinson's Progression Markers, Association of cerebrospinal fluid beta-amyloid 1-42, T-tau, P-tau181, and alphasynuclein levels with clinical features of drug-naive patients with early Parkinson disease, JAMA Neurol 70(10) (2013) 1277-87.

[24] Y. Compta, T. Valente, J. Saura, B. Segura, A. Iranzo, M. Serradell, C. Junque, E. Tolosa, F. Valldeoriola, E. Munoz, J. Santamaria, A. Camara, M. Fernandez, J. Fortea, M. Buongiorno, J.L. Molinuevo, N. Bargallo, M.J. Marti, Correlates of cerebrospinal fluid levels of oligomeric- and totalalpha-synuclein in premotor, motor and dementia stages of Parkinson's disease, Journal of neurology 262(2) (2015) 294-306.

[25] F. Llorens, N. Kruse, M. Schmitz, N. Gotzmann, E. Golanska, K. Thune, O. Zejneli, E. Kanata, T. Knipper, M. Cramm, P. Lange, S. Zafar, B. Sikorska, P.P. Liberski, E. Mitrova, D. Varges, C. Schmidt, T. Sklaviadis, B. Mollenhauer, I. Zerr, Evaluation of alpha-synuclein as a novel cerebrospinal fluid biomarker in different forms of prion diseases, Alzheimer's \& dementia : the journal of the Alzheimer's Association (2016).

[26] B. Mollenhauer, J.J. Locascio, W. Schulz-Schaeffer, F. Sixel-Döring, C. Trenkwalder, M.G. Schlossmacher, $\alpha$-Synuclein and tau concentrations in cerebrospinal fluid of patients presenting with parkinsonism: a cohort study, The Lancet Neurology 10(3) (2011) 230-240.

[27] S. Hall, A. Ohrfelt, R. Constantinescu, U. Andreasson, Y. Surova, F. Bostrom, C. Nilsson, W. Hakan, H. Decraemer, K. Nagga, L. Minthon, E. Londos, E. Vanmechelen, B. Holmberg, H. Zetterberg, K. Blennow, O. Hansson, Accuracy of a panel of 5 cerebrospinal fluid biomarkers in the differential diagnosis of patients with dementia and/or parkinsonian disorders, Arch Neurol 69(11) (2012) 1445-52.

[28] B. Mollenhauer, V. Cullen, I. Kahn, B. Krastins, T.F. Outeiro, I. Pepivani, J. Ng, W. SchulzSchaeffer, H.A. Kretzschmar, P.J. McLean, C. Trenkwalder, D.A. Sarracino, J.P. Vonsattel, J.J. Locascio, O.M. El-Agnaf, M.G. Schlossmacher, Direct quantification of CSF alpha-synuclein by ELISA and first cross-sectional study in patients with neurodegeneration, Exp Neurol 213(2) (2008) $315-25$.

[29] P. Oeckl, F. Metzger, M. Nagl, C.A. von Arnim, S. Halbgebauer, P. Steinacker, A.C. Ludolph, M. Otto, Alpha-, Beta-, and Gamma-synuclein Quantification in Cerebrospinal Fluid by Multiple Reaction Monitoring Reveals Increased Concentrations in Alzheimer's and Creutzfeldt-Jakob Disease but No Alteration in Synucleinopathies, Molecular \& cellular proteomics : MCP 15(10) (2016) 3126-3138.

[30] N.K. Majbour, N.N. Vaikath, K.D. van Dijk, M.T. Ardah, S. Varghese, L.B. Vesterager, L.P. Montezinho, S. Poole, B. Safieh-Garabedian, T. Tokuda, C.E. Teunissen, H.W. Berendse, W.D. van de Berg, O.M. El-Agnaf, Oligomeric and phosphorylated alpha-synuclein as potential CSF biomarkers for Parkinson's disease, Mol Neurodegener 11 (2016) 7. 